transplant patients with immunosuppressive treatment. In only 2 cases there was a previous arthrocentesis. The mean diagnostic delay was 14 days (SD 13) (data available in 14 cases). The most commonly affected joint was the knee (48\%), followed by the foot $(20 \%)$ and the hip (12\%). In 2 cases several joints were involved at the same time. In synovial fluid cytological studies, the most frequently identified crystals were: urate $(60 \%)$, calcium pyrophosphate $(20 \%)$ and hydroxyapatite ( $8 \%$ ). In $32 \%$ of cases gram staining was positive, but $88 \%$ of patients had a positive joint fluid culture, with the most frequently isolated germs being methicillin-sensitive $S$. aureus (48\%), methicillin-resistant $S$. aureus (MRSA) (12\%) and M. tuberculosis (12\%). $32 \%$ of patients presented positive blood cultures ( $12 \%$ with negative synovial fluid culture), although $48 \%$ of patients had fever at the time of diagnosis. It should be noted that $48 \%$ had radiological baseline damage. Surgical debridement was performed in $32 \%$ of patients. Evolution was successful in $56 \%$ of patients; although intercurrent complications were usual $(40 \%)$. Mortality was $8 \%$-one case due to acute pulmonary edema and the other because of septic shock.

Conclusions: Coexistence of infectious and CA represents a diagnostic challenge and requires a high suspicion index. It usually appears in elderly patients with comorbidities. Gout was the most prevalent CA. S. aureus was the most commonly causative pathogen, with a high rate of MRSA infection. If it's treated early the evolution is usually favorable, which makes synovial fluid microbiological study imperative.

Disclosure of Interest: None declared

DOI: 10.1136/annrheumdis-2017-eular.4993

\section{SAT0561 USEFULNESS OF POLYMERASE CHAIN REACTION FOR DIAGNOSING WHIPPLE'S DISEASE IN RHEUMATOLOGY}

M. Herbette ${ }^{1}$, J.B. Cren ${ }^{2}$, L. Joffres ${ }^{3}$, C. Lucas ${ }^{4}$, E. Ricard ${ }^{5}$, C. Salliot ${ }^{6}$, J. Guinard ${ }^{5}$, A. Perdriger ${ }^{4}$, E. Solau-Gervais ${ }^{3}$, B. Bouvard ${ }^{2}$, A. Saraux ${ }^{1}$ on behalf of Reseau Victor Hugo. ${ }^{1}$ Rheumatology, $\mathrm{CHU}$ Brest and Université Bretagne Occidentale, Brest; ${ }^{2}$ Rheumatology, CHU, Angers; ${ }^{3}$ Rheumatology, $\mathrm{CHU}$, Poitiers; ${ }^{4}$ Rheumatology, $\mathrm{CHU}$, Rennes; ${ }^{5}$ Rheumatology; ${ }^{6} \mathrm{CHU}$, Orleans, France

Background: No consensus exists about the combination of clinical, laboratory, and radiological findings that warrant tests for Whipple's disease.

Objectives: The primary aim of this multicentre retrospective study was to determine when patients evaluated for rheumatological symptoms should undergo T. whipplei PCR testing. Secondary aims were to describe the clinical patterns and treatments used, to determine the diagnostic yield of PCR testing, and to assess whether centres with higher numbers of tests also had a larger number of Whipple's disease diagnoses.

Methods: In a retrospective observational study done in five hospitals, we assessed the clinical and radiological signs that prompted T. whipplei PCR testing between 2010 and 2014, the proportion of patients diagnosed with Whipple's disease, the number of tests performed and the number of diagnoses according to the number of tests, the patterns of Whipple's disease, and the treatments used. Results: At least one PCR test was performed in each of 267 patients. Rheumatic signs were peripheral arthralgia $(n=239,89 \%)$, peripheral arthritis $(n=173,65 \%)$, and inflammatory back pain $(n=85,32 \%)$. The main extra-articular signs were constitutional symptoms $(n=111,41.8 \%)$, diarrhoea $(n=70,26.5 \%)$, fever $(n=53$, $20 \%)$, lymphadenopathy $(n=14,5.3 \%)$, and neurological signs $(n=11,4.2 \%)$. Whipple's disease was diagnosed in 13 patients $(4.9 \%)$. The main samples tested and the more frequently positive tests in the centres with diagnoses of Whipple's disease were saliva and stool. In the centres with no diagnoses of Whipple's disease, arthritis was less common, whereas constitutional symptoms, fever, and lymphadenopathy were more common. 11 patients with Whipple's disease had CRP elevation. The annual incidence ranged across centres from 0 to $3.6 / 100000$ inhabitants. The patients group with Whipple's disease had a higher proportion of males, older age, and greater frequency of arthritis.

Conclusions: Males aged $40-75$ years with unexplained intermittent seronegative peripheral polyarthritis, including those without constitutional symptoms, should have T. whipplei PCR tests on saliva, stool and, if possible, joint fluid.

Disclosure of Interest: None declared

DOI: 10.1136/annrheumdis-2017-eular.5852

\section{SAT0562 CHIKUNGUNYA FEVER IN PATIENTS WITH PRIOR RHEUMATIC DISEASES: IS IT MORE SEVERE?}

A.T. Dantas ${ }^{1}$, M.S. Luna ${ }^{1}$, P.R. Santos ${ }^{1}$, C.A. Andrade ${ }^{1}$, C.D.L. Marques ${ }^{1}$, A. Ranzolin ${ }^{2}$, N.G. Cavalcanti ${ }^{1}$, R.S. Gonçalves ${ }^{3}$, L.F. Rocha Junior ${ }^{2}$, H.D. Lima ${ }^{2}$, P.R. Melo ${ }^{3}$, L.D. Valadares ${ }^{4}$, C.A. da Fonte ${ }^{4}$, M.L.D. Valadares ${ }^{4}$ E. Freire ${ }^{5}$, A.K.G. Melo ${ }^{5}$, M.M. Medeiros ${ }^{6}$, M.C. Bezerra ${ }^{6}$, V.B. Marques ${ }^{6}$, R.A. Océa ${ }^{7}$, A.L. Duarte ${ }^{1} .{ }^{1}$ Internal Medicine, Universidade Federal de Pernambuco; ${ }^{2}$ Rheumatology; ${ }^{3}$ Internal Medicine, Instituto de Medicina Integral Prof. Fernando Figueira; ${ }^{4}$ Rheumatology, Hospital Getulio Vargas, Recife; ${ }^{5}$ Rheumatology, Universidade Federal da Paraíba, João Pessoa;

${ }^{6}$ Rheumatology, Universidade Federal do Ceará, Fortaleza: ${ }^{7}$ Rheumatology, Universidade Federal de Sergipe, Aracaju, Brazil

Background: Chikungunya fever (CF) is an infectious disease caused by a RNA virus and its transmission occurs by the inoculation of the virus by the female bite of Aedes aegypti mosquito. In Brazil, where the vetor is endemic, the virus rapidly disseminated and there was an epidemic, specially in the Northeast region of the country with 263.980 notified cases in 2016 . It is known that CF may have a chronic course with articular symptons, however there is not consistent data in the medical literature on CF evolution in patients with prior rheumatic diseases. Objectives: To assess whether there is any difference in the characteristics of articular manifestations of $\mathrm{CF}$ in patients with prior inflammatory rheumatic diseases (IRD), non-inflammatory rheumatic diseases (NIRD) and controls (patients with no diagnosed prior rheumatic diseases).

Methods: Cross-sectional study using a database from CHIKBRASIL cohort. Patients enrolled had clinical and epidemiological characteristics of CF and were classified in three groups: IRD (rheumatoid arthritis, axial spondyloarthritis and systemic lupus erythematosus), NIRD (fibromyalgia and osteoarthritis) and controls (no prior rheumatic diseases).

Results: A total of 150 patients were enrolled. There were 55 patients with IRD, 40 patients with NIRD and 55 controls, paired by age and sex. There were no differences in acute phase symptoms in the groups. There a was more frequent occurrence of arthritis in patients with IRD compared to NIRD ( $p=0.001)$ and to controls $(p=0.002)$. In $89.1 \%$ of the patients with IRD there was an underlying disease exacerbation and $74 \%$ described an expressive worsening of symptoms compared to the period prior to infection. Patients with IRD had an increase in the current dose of corticosteroids (median 10mg, IQR 10-20) compared to previous dose used (median 6mg, IQR 5-10) after the onset of CF ( $p=0.0007)$. Importantly, there was more methotrexate prescription $(23.5 \%)$ in IRD group, compared to NIRD group ( $0, p-0.001)$ and to controls $(3.7 \%, p=0.003)$.

Conclusions: Patients with IRD and CF presented significantly more arthritis compared to NIRD or to controls. CF seems to induce underlying rheumatologic disease exacerbation in patients with inflammatory disease and a more aggressive therapeutic approach might be necessary in this group of patients.

Disclosure of Interest: None declared

DOI: 10.1136/annrheumdis-2017-eular.6560

\section{SAT0563 EVALUATION OF ROLE OF SLEEP DISTURBANCE, DEPRESSION, OBESITY, AND PHYSICAL INACTIVITY IN FATIGUE IN CHIKUNGUNYA ARTHRITIS}

\section{Bhama. Orthopedics, Sardar Patel Medical College, Bikaner, India}

Background: Chikungunya virus (CHIKV) is a mosquito-borne alphavirus that circulates predominantly in tropical and subtropical regions, potentially affecting over 1 billion people.

Objectives: Fatigue is a major concern in individuals where $\mathrm{CHIKV}$ results severe chronic arthralgia and/or arthritis lasting months to years. However, in order to treat fatigue adequately, its sources need to be identified.

Methods: All participants had physician-diagnosed CHIKV arthralgia. Data regarding self-reported sleep quality, depression, physical activity, disease activity, muscle strength, functional limitations, and body composition were collected during a single home visit (number of participants $=117$ ). Information on demographics, medications, alcohol intake and smoking was collected. The Fatigue Severity Inventory (FSI; measuring average fatigue over the past 7 days) was used as the primary outcome. Analyses were conducted to evaluate bivariate relationships with fatigue and correlations among risk factors. Multivariate analyses identified independent predictors of fatigue.

Results: The mean age was $48 \pm 16$ years, the mean disease duration was $7 \pm 6$ years, and $71 \%(\mathrm{M}: 33, \mathrm{~F}: 84)$ of subjects were female. The mean $\mathrm{FSI}$ rating was $4.1 \pm 2.0$ (range $0-10$ ). In multivariate analyses, self-reported disease activity, poor sleep, depression, and obesity were independently associated with fatigue. Physical inactivity was correlated with poor sleep, depression, and obesity. Mediation analyses indicated that physical inactivity had an indirect association with fatigue, mediated by poor sleep, depression, and obesity.

Conclusions: This cross-sectional study suggests that fatigue may not be solely a result of CHIKV arthralgia, but may result from a constellation of factors that includes CHIKV arthralgia disease activity or pain, but also includes inactivity, depression, obesity, and poor sleep. The results suggest need of various interventions to improve fatigue in individuals with CHIKV arthritis, including increasing physical activity or addressing depression or obesity.

\section{References:}

[1] Thiberville S.D., Moyen N., Dupuis-Maguiraga L., Nougairede A., Gould E.A., Roques P., de Lamballerie X. Chikungunya fever: epidemiology, clinical syndrome, pathogenesis and therapy. Antiviral Res. 2013;99(3):345-370.

[2] Vijayan V, Sukumaran S. Chikungunya Virus Disease: An Emerging Challenge for the Rheumatologist. J Clin Rheumatol. 2016 Jun;22(4):203-11.

[3] Stebbings S, Herbison P, Doyle T, Treharne GJ, Highton J. A comparison of fatigue correlates in rheumatoid arthritis and osteoarthritis: disparity in associations with disability, anxiety, and sleep disturbance. Rheumatology (Oxford)2010:49:361-7.

[4] Whitehead L. The measurement of fatigue in chronic illness: a systematic review of unidimensional and multidimensional fatigue measures. J Pain Symptom Manage2009;37:107-28.

Disclosure of Interest: M. Bhama Consultant for: Orthopedics Consultant DOI: 10.1136/annrheumdis-2017-eular.6670 